Relations industrielles

Industrial Relations

\title{
Richard HYMAN, Wolfgang STREECK : New Technology and Industrial Relations. Oxford, Basil Blackwell, 1988, 309 pp., ISBN 0-631-15982-7
}

\section{Jacques Bélanger}

Volume 45, numéro 1, 1990

URI : https://id.erudit.org/iderudit/050574ar

DOI : https://doi.org/10.7202/050574ar

Aller au sommaire du numéro

Éditeur(s)

Département des relations industrielles de l'Université Laval

ISSN

0034-379X (imprimé)

1703-8138 (numérique)

Découvrir la revue

Citer ce compte rendu

Bélanger, J. (1990). Compte rendu de [Richard HYMAN, Wolfgang STREECK : New Technology and Industrial Relations. Oxford, Basil Blackwell, 1988, 309 pp., ISBN 0-631-15982-7]. Relations industrielles / Industrial Relations, 45(1),

208-209. https://doi.org/10.7202/050574ar

Tous droits réservés @ Département des relations industrielles de l'Université Laval, 1990
Ce document est protégé par la loi sur le droit d'auteur. L'utilisation des services d’Érudit (y compris la reproduction) est assujettie à sa politique d'utilisation que vous pouvez consulter en ligne.

https://apropos.erudit.org/fr/usagers/politique-dutilisation/ 
Même si la problématique soulevée par Heckscher s'inspire de la situation américaine, elle conserve une pertinence certaine dans le contexte canadien. Notre régime de relations du travail reprend les principes qui fondent le Wagner Act. Les syndicats canadiens ne subissent pas la crise de leurs confrères américains à cause de l'essor très important des syndicats canadiens dans les services publics. Toutefois, comme leurs collègues américains, les organisations syndicales canadiennes ne réussissent pas de percée significative dans les services privés. Les entreprises canadiennes ont à relever le défi de la concurrence mondiale. Les syndicats doivent de plus en plus être conscients de l'interdépendance entre d'une part, la prospérité de l'entreprise et d'autre part, le développement des emplois et l'amélioration des conditions de travail.

Heckscher ne prétend pas se fonder sur des études empiriques et sa «méthodologie» demeure intuitive et impressionniste. L'auteur manifeste, au niveau de l'examen de la problématique, un esprit de synthèse impressionnant lorsqu'il dégage les tendances historiques développées à partir du Wagner Act. Les solutions avancées par Heckscher visent à provoquer la réflexion et sont peut-être à la mesure de la crise syndicale aux Etats-Unis. L'auteur donne plutôt l'impression qu'il cherche à secouer les syndicalistes américains pour les sortir de leur torpeur et de leur conservatisme.

Le lecteur trouvera difficilement importable les remèdes prescrits par Heckscher. Il les trouvera même irréalistes. Toutefois, le sentiment d'urgence qui se dégage de ce volume aurait avantage à se communiquer aux syndicalistes canadiens pour qu'ils développent des stratégies adaptées aux défis des années 2000 et pour qu'ils n'attendent pas d'être confrontés à une remise en question de leur pertinence.

Michel GRANT

Université du Québec à Montréal

New Technology and Industrial Relations, by Richard Hyman and Wolfgang Streeck (eds.), Oxford, Basil Blackwell, 1988, 309 pp., ISBN 0-631-15982-7

Cet ouvrage de la collection Warwick Studies in Industrial Relations regroupe des textes originaux qui ont d'abord été présentés en 1986 à l'occasion d'un colloque international organisé par Richard Hyman et Wolfgang Streeck. Le caractère international de l'ouvrage est bien senti, puisque les auteurs des dix-huit chapitres proviennent d'une dizaine de pays, principalement d'Europe de l'Ouest. Une introduction assez substantielle expose la structure du volume en cinq parties, de même que les thèmes principaux de chaque contribution. J'ai choisi de faire état des chapitres qui paraissent d'un apport plus durable et de ceux qui devraient intéresser particulièrement les lecteurs de Relations industrielles.

Le texte de A. Sorge et W. Streeck intitulé «Industrial Relations and Technical Change: The Case for an Extended Perspectiven apporte une contribution certaine à la littérature. J'en retiens trois idées principales. Ils soulignent d'abord le traitement inadéquat du changement technologique par l'approche institutionnaliste en relations industrielles, laquelle conçoit la technologie comme un facteur exogène. Ils abordent aussi, de façon très stimulante, l'interaction complexe et encore peu explorée entre l'organisation du travail et le cadre institutionnel des relations du travail. Enfin, ils s'insèrent dans le débat sur les stratégies industrielles en proposant une classification en quatre types. Ils favorisent clairement le type appelé diversified quality production, qui s'apparente au concept de spécialisation flexible de Piore et Sabel.

Cette question de la flexibilité et du déclin possible du fordisme constitue de fait l'un des principaux thèmes du volume. Le texte de Richard Hyman porte précisément sur une analyse 
de la thèse de Piore et Sabel et en constitue à ma connaissance la critique la plus articulée. Néanmoins, Hyman donne en fait beaucoup de crédit à cette thèse et ne cherche pas à clore le débat. Il souligne notamment en quoi les contradictions inhérentes à la fonction managériale affectent la diffusion et la cohérence de stratégies industrielles aussi globales que le fordisme ou la spécialisation flexible. Cette position semble partagée par Stephen Wood, qui insiste beaucoup sur le fait que la compréhension des développements en cours dans l'industrie américaine de l'automobile commande une analyse plus nuancée. Sur la base d'un programme d'entrevues réalisées aux Etats-Unis en 1985 (voir aussi son article dans Economic and Industrial Democracy, 1986), Wood soutient que la tendance observée ne correspond ni à un stéréotype du fordisme ni à celui de la spécialisation flexible. Bien qu'il n'aille pas jusqu'à proclamer les vertus de l'empirisme pur, il faut reconnaître que son souci d'adapter une position nuancée affecte en plusieurs endroits la clarté de l'exposé.

Parmi ces textes qu'il faudra garder en mémoire, il convient d'insister sur celui de Peter Armstrong intitulé «Labour and Monopoly Capital». Comme on l'aura deviné, ce chapitre porte sur la thèse de Braverman, dont il fait la défense. Compte tenu que la critique (souvent précédée d'un hommage) de cet auteur est devenue un lieu commun, ce chapitre est très pertinent en ce qu'il suggère un retour sur la démarche initiale. Après avoir souligné que les études détaillées sur les effets de la microélectronique ne sont pas unanimes à rejeter la thèse de la déqualification, Armstrong insiste beaucoup sur le fait que l'analyse de Braverman se situait à un niveau beaucoup plus général, en longue période. Il percevait alors une tendance générale à la déqualification, entendue surtout comme la séparation entre la conception et l'exécution, dans le mode de production capitaliste.

Soulignons enfin le chapitre de Harry Katz intitulé «Policy Debates over Work Reorganization in North American Unions». Ce texte est quelque peu décevant. La discussion se développe à partir d'une distinction entre les approches qu'il qualifie de "coopérative» et de «militante» à l'égard des changements à l'organisation du travail. Malgré la simplicité et l'attrait possible de la distinction, le lecteur n'est pas convaincu de son pouvoir explicatif. La méthode repose beaucoup sur le discours de quelques leaders nationaux et ne permet pas d'explorer le processus démocratique au sein des organisations. Elle n'incite pas davantage à considérer les tensions inévitables dans la formulation des politiques syndicales sur une question aussi complexe que la réorganisation du travail. Le lecteur canadien notera aussi que, malgré le titre du chapitre, la démarche ne permet pas d'aborder sérieusement le caractère distinct des politiques syndicales canadiennes.

En somme, il s'agit d'un ouvrage important qui s'impose par la qualité et la pertinence de plusieurs de ses composantes. Il comporte néanmoins certaines des limites assez généralement associées aux publications issues de colloques. Quelques chapitres ne portent pas vraiment sur les nouvelles technologies. Les responsables de la collection avancent que le fil conducteur du volume est le rejet du déterminisme technologique. À ce stade-ci, cette position ne peut plus être davantage qu'un point de départ. Comme le montre aussi la sociologie française, il s'agit maintenant de préciser davantage l'articulation entre technologie et organisation, considérant les contraintes générées par une technologie spécifique aussi bien que les options ouvertes aux acteurs dans l'entreprise.

Jacques BÉLANGER

Université Laval 